\title{
Failure Mechanism Analysis of Elevator Brake
}

\author{
Xiao Liang \\ Shanghai Institute of Special Equipment Inspection and Technical Research \\ SSEI \\ Shanghai, China \\ liangxiao@ssei.cn
}

Keywords: elevator accidents;brake; working principle; plunger damping stuck fault;preventive measures

\begin{abstract}
With the constant improvement of urban construction ,the elevator has become an indispensable part of the modern city.In recent years ,elevator accidents often occurred .The main factor of accidents was the fault of brake. The elevator brake is an important part of the elevator traction machine. The basic function of the brake is to keep the static state of the elevator car when the elevator stops. When the elevator fails, the car can decelerate and keep the car in an emergency state. This article mainly demonstrates the role and importance of elevator brake ,based on the structure and working principle of elevator electromagnetic brake and work characteristics . At the same time, combined with an elevator brake plunger damping stuck fault, analyses the fault of the plunger, magnetic ring, loose gate and other related parts and puts forward preventive measures.
\end{abstract}

\section{Introduction}

With the constant improvement of urban construction, the elevator has become an indispensable part of the modern city.In recent years, elevator accidents often occurred .The main factor of accidents was the fault of brake. The elevator brake is an important part of the elevator traction machine. It is a kind of braking device which uses spring pressure to produce resistance braking and uses electric power to release. This article combined with an elevator brake plunger damping stuck fault, analyses the fault of the plunger, magnetic ring, loose gate and other related parts and put forward preventive measures.

\section{Brake mechanical structure}

The brakes used on the elevator equipment are friction-type electromechanical "normally closed" brakes. The brake mechanical structure mainly consists of four parts: directed pressure spring that produces power; electromagnet device that produces release force; Brake pad applied to braking on the brake wheel; brake arm, drive and adjustment mechanism.

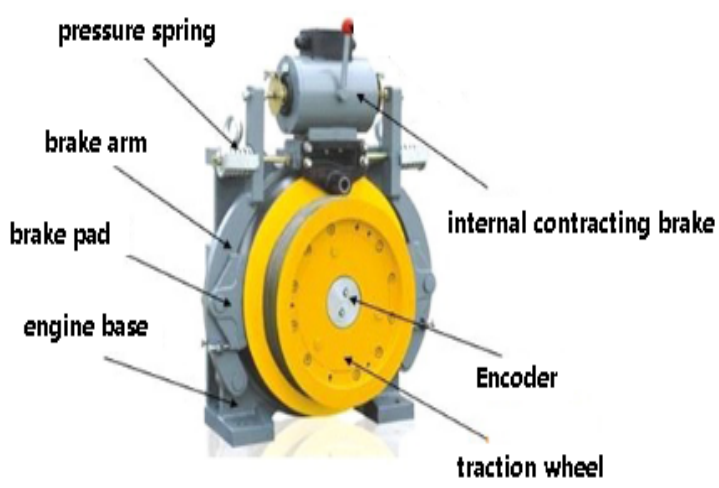

Figure 1. Brake mechanical structure 


\section{Brake working principle}

The brake consists of two working conditions:lock and release brake.It is controlled by the elevator electromagnetic coil energizing or lossing power. When the electromagnet coil is energized, electromagnetic plunger in the current magnetic field is rapidly magnetized,between the plunger pull each other under the action of magnetism,thus driving the brake arm to overcome the brake pressure spring force.When the electromagnet coil is out of power,there is no attraction between electromagnetic plungers,the brake pressure spring is in the release state,the brake pad is uniformly pressed on the brake wheel under the action of the brake pressure spring,hold the brake wheel tightly.

\section{The case of plunger damping failure}

One day,the elevator hoisting accident occurred in a residential area.Before the accident,the elevator car opened at the first floor, and suddenly ran up until it hit the top of the well.One passengers were injured in this accident.Via checking, the plunger damping failure of the elevator brake is the direct cause of the accident.

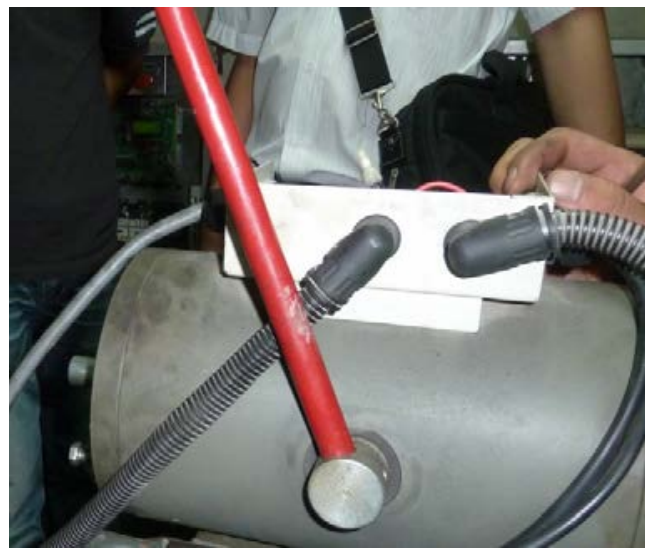

Figure 2. Invalid elevator brake

\section{A. Remove detection}

After the brake is disassembled,cut the frame and guide ring (sleeve) lengthwise along the center,visible plunger and guide ring (set) have obvious longitudinal wear and tear striation.In the gap region, there is a small powder and a sheet of adhesive.These particles and adhesion are formed along the magnetic field lines in the magnetic field.All of them are ferromagnetic materials.

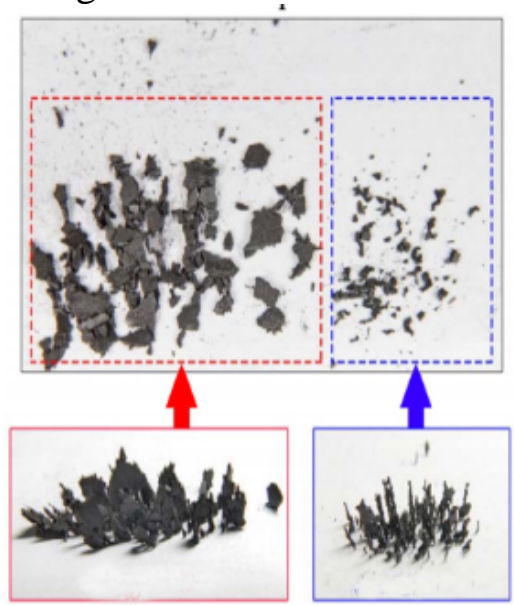

Figure 3. Morphology under magnetic field

\section{B. Electron microscope and $X$ ray energy spectrum analysis of particles}

The morphology of the adhesive under electron microscope is shown in Figure 4, showing polygonal, blocky and powder morphology.different regions of these blocks were analyzed by $\mathrm{X}$ ray 
energy spectrum .The main elements and reference content are shown in table 1.

TABLE I. MAIN ELEMENTS AND REFERENCE CONTENT OF SMALL PARTICLES (POWDER)

\begin{tabular}{|c|c|c|c|c|c|c|}
\hline Main elements & $\mathrm{O}$ & $\mathrm{Si}$ & $\mathrm{S}$ & $\mathrm{Mn}$ & $\mathrm{Zn}$ & $\mathrm{Fe}$ \\
\hline Area 1 & 21.8 & 5.0 & 0.68 & 0.34 & 1.6 & 70.4 \\
\hline Area 2 & 21.5 & 5.0 & 0.68 & 0.37 & 1.5 & 70.0 \\
\hline Area 3 & 26.4 & 5.5 & 1.0 & $/$ & 1.2 & 64.5 \\
\hline
\end{tabular}

\section{Comprehensive analysis of Plunger}

Two iron core shape and damage morphology are basically the same.In the two iron core and the magnetic cover (ring) to match the segment has obvious wear phenomenon,its matching segment traces width of $22.2 \mathrm{~mm} \sim 23.2 \mathrm{~mm}$.

In the bottom surface of the two plunger body, there are a number of impact indentation with the loose bars, the depth of the marks is different, and the fan distribution shows that the plunger has a certain rotational motion in the operation.
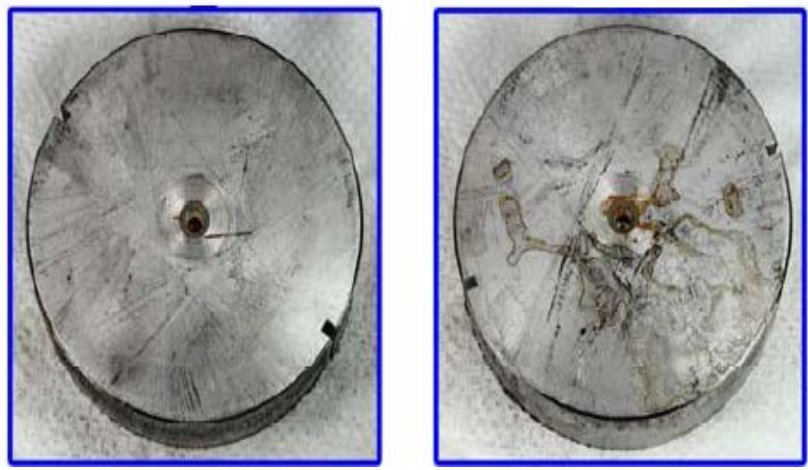

Figure 4. Plunger damage morphology

Metallographic analysis was performed on the cross section of the wear zone.In the cross-section, we can see that the surface of the worn surface is in the form of a depression, the flow rate is as deep as $0.024 \mathrm{~mm}$ and the matrix structure is iron element $+\mathrm{a}$ small amount of pearlite,And can be seen folded rheological cracking morphology, rheological depth of $0.08 \mathrm{~mm}$.Distribution of cracks in the gray oxide, indicating that the late surface oxidation due to rheological rheology.

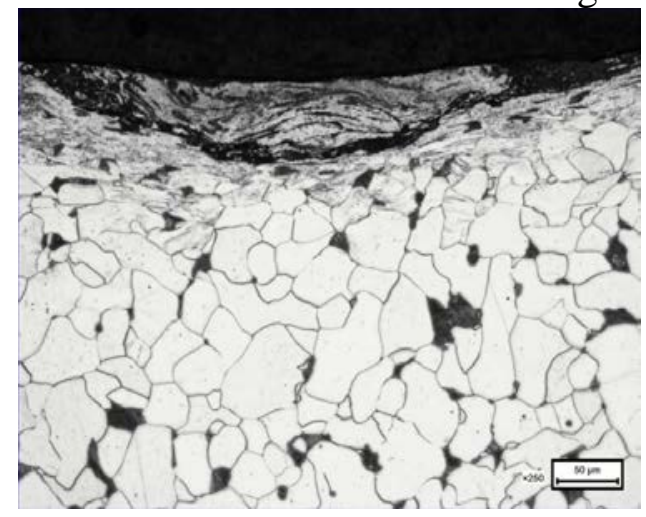

Figure 5. Worn surface structure is subject to extrusion rheology

D. Comprehensive analysis of magnetic guide ring (set)

As shown in figure 6, the outer diameter of the guide ring is about phi $42 \mathrm{~mm}$. The width is about 
$67 \mathrm{~mm}$; The outer diameter of the guide sleeve is approximately phi $102 \mathrm{~mm}$ and the width is about $5 \mathrm{~mm}$.

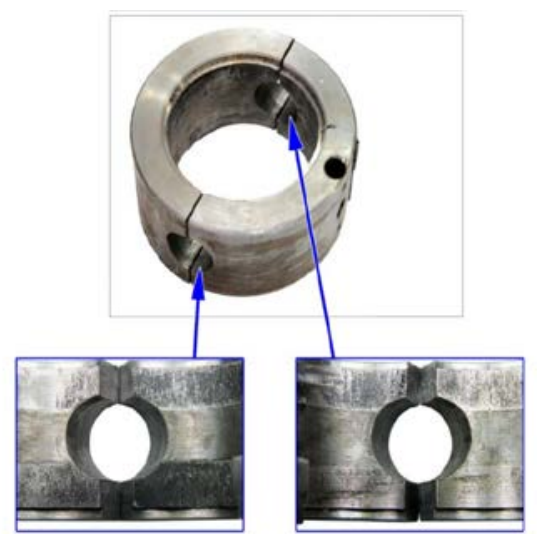

Figure 6. Magnetic ring wear morphology

The inner surface of the two magnetic guide sleeve is blocked by the uneven wear. The wear area basically corresponds to the inner surface of the permeable ring, the shape of the furrow is worn, and the shades are different in depth. However, there is no obvious wear in some areas, and ring marks can still be seen.

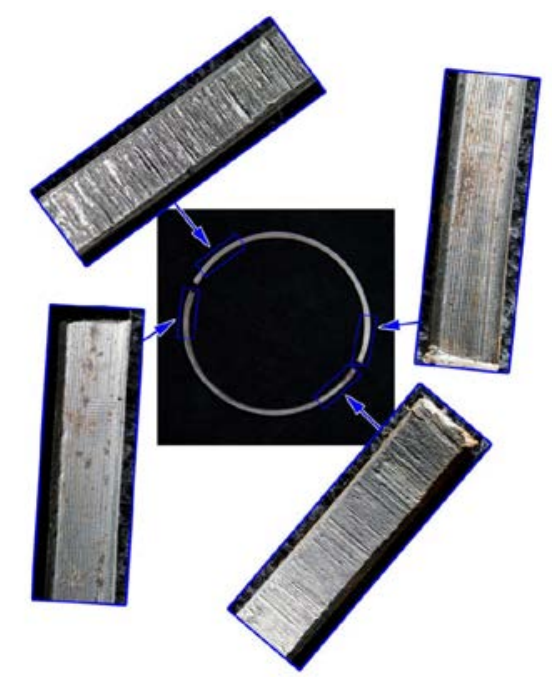

Figure 7. Magnetic sleeve and wear morphology

\section{E. Comprehensive analysis of slack brake lever}

The outer circle of the slack brake lever is about $33.5 \mathrm{~mm}$, and the length is about $191 \mathrm{~mm}$. The material is cold drawn 45 steel, and the outer surface is galvanized Daniel.

The fitting section of the slack brake lever and the magnetic guide ring is badly worn, and it is visible to the furrow of the furrowThe diameter of the section is approximately phi $33.07 \mathrm{~mm} \sim$ phi $33.10 \mathrm{~mm}$, and the outer diameter of the unworn segment is approximately phi $33.24 \mathrm{~mm}$, indicating the wear of about $0.07 \mathrm{~mm}$ (unilateral), as shown in figure 8 . 


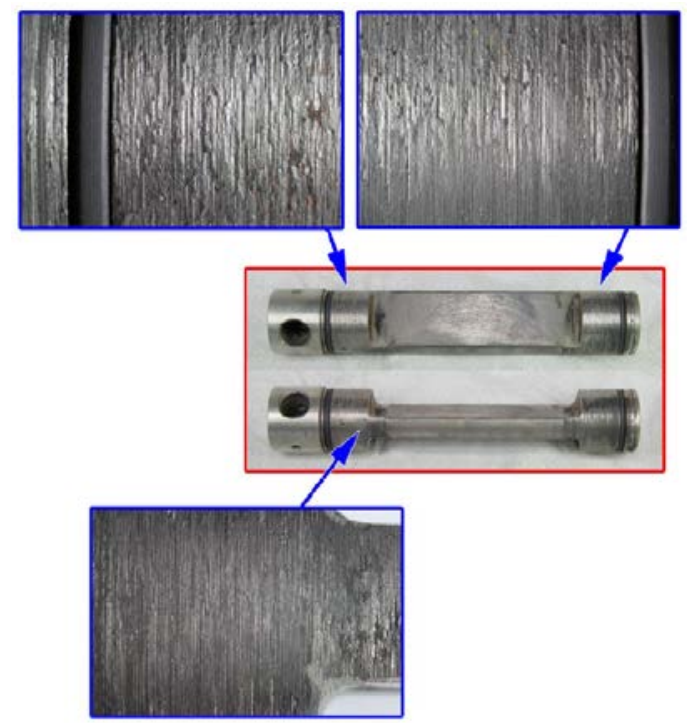

Figure 8. Slack brake lever wear morphology

\section{Failure analysis conclusion}

Based on the above analysis results, it can be concluded that the failure of plunger damping card is related to the abnormal adhesion of plunger and magnetic ring.This adhesive wear occurs mainly due to the abnormal rotation of the slack brake lever and the formation of the embedded plunger and magnetic ring clearance.

\section{Preventive measure}

As the saying goes, "elevator safety third by product, seven by maintenance".As the elevator manufacturing unit,strict quality control for some key parts, ensure that the elevator is in accordance with safety technical specifications and national standards, and is responsible for the safety performance of elevators.As an elevator maintenance unit,regular cleaning, lubrication, inspection and adjustment of the brake,avoiding accidents caused by elevator maintenance and maintenance.

\section{References}

[1] M. Kumbhar, A. Walavalkar, Magnetostatic analysis and power optimization ofelectric release brake, IEEE International Conference on Magnetics, Machines\& Drives (AICERA-2014 iCMMD) (2014).

[2] R.J. Farris, M. Goldfarb, Design of a multidisc electromechanical brake,IEEE/ASME Trans. Mechatron. 16 (6) (2011) 985-993.

[3] Kesheng Wang,Bing Lei. Using B-spline neural network to extract fuzzy rules for a centrifugal pump monitoring[J]. Journal of Intelligent Manufacturing . 2001 (1).

[4] [2]Seiji Watanabe,Takashi Yumura,Yoshikatsu Hayashi,and Yukihiro Takigawa.Development of Elevator Simulator for Emergent Behavior. The 10 (th) Transportation and Logistics Conference . 2001.

[5] [3]FENG Yonghui ZHANG Jianwu School of Mechanical Engineering,Shanghai Jiaotong University,Shanghai 200240,China. HYDRAULIC ACTIVE GUIDE ROLLER SYSTEM FOR HIGH-SPEED ELEVATOR BASED ON FUZZY CONTROLLER[J]. Chinese Journal of Mechanical Engineering. 2007(05). 